\section{Istmectomía laparoscópica asistida por robot en un paciente con ectopia renal cruzada fusionada por un tumor renal}

López-Verdugo JF, ${ }^{1}$ Briseño-Rentería JI, ${ }^{2}$ Estrada-Bujanos JM, ${ }^{3}$ Camacho-Trejo VF, ${ }^{3}$ Castro-Zazueta $S,{ }^{4}$ Quintero-Badillo JE, ${ }^{2}$ y colaboradores.

\section{Resumen}

ANTECEDENTES: la localización anómala del riñón en el espacio retroperitoneal se denomina ectopia renal. La ectopia renal cruzada constituye una malformación congénita excepcional con incidencia de 1 caso por cada 1350 a 1 por cada 2000 nacimientos. El tumor más común reportado en alteraciones de la fusión renal es el carcinoma de células renales. Las anomalías de la fusión renal aumentan cuatro veces el riesgo de neoplasias.

CASO CLÍNICO: paciente masculino de 64 años de edad con antecedentes oncológicos de ectopia renal y pulmonar. En los estudios de rutina se detectó una masa en la fusión de ambos riñones. Se realizó istmectomía con plastia ureteral, con colgajo de Scardino y colocación de catéter doble J mediante laparoscopia asistida por robot.

CONCLUSIÓN: la ectopia renal fusionada cruzada se origina por el desarrollo anormal de la yema ureteral y el blastema metanéfrico durante la cuarta y octava semanas del embarazo. Después del riñón en herradura, la ectopia renal cruzada fusionada es la anormalidad renal más frecuente. La ectopia renal cruzada es una alteración congénita poco frecuente; el cruce de izquierda a derecha es frecuente, contrario al caso aquí reportado. La relación de tumores malignos con estas anomalías representa un evento excepcional. La anatomía es impredecible, se observan anomalías vasculares en $70 \%$ de los casos lo que complica el manejo quirúrgico. Diversos estudios han intentado establecer el origen del fenotipo sarcomatoide y su relación con cualquiera de los patrones histológicos clásicos del carcinoma renal.

PALABRAS CLAVE: ectopia renal cruzada, ismectomía y tumor renal.

\footnotetext{
${ }^{1}$ Director.

${ }^{2}$ Fellowship en Endourología y Laparoscopia Avanzada.

${ }^{3}$ Urólogo.

${ }^{4}$ Fellowship en Cirugía Robótica.

Instituto de Urología Robótica y Laparoscópica, Nuevo León, Monterrey.
}

Recibido: septiembre 2017

Aceptado: octubre 2017

Correspondencia

Dr. Juan Iván Briseño Rentería

dr_briseno@hotmail.com

Este artículo debe citarse como López-Verdugo JF, Briseño-Rentería Jl, EstradaBujanos JM, Camacho-Trejo VF, Castro-Zazueta S, Quintero-Badillo JE, y col. Istmectomía laparoscópica asistida por robot en un paciente con ectopia renal cruzada fusionada por un tumor renal. Rev Mex Urol. 2017 nov-dic;77(6):470-474.

DOI: https://doi.org/10.24245/revmexurol.v77i6.1653 


\section{Robotic-assisted laparoscopic isthmusectomy in crossed-fused renal ectopia due to kidney tumor}

\author{
López-Verdugo JF, ${ }^{1}$ Briseño-Rentería JI, ${ }^{2}$ Estrada-Bujanos JM, ${ }^{3}$ Camacho-Trejo
} $\mathrm{VF}^{3}{ }^{3}$ Castro-Zazueta $\mathrm{S},{ }^{4}$ Quintero-Badillo JE, ${ }^{2}$ et al.

\begin{abstract}
BACKGROUND: Anomalous kidney location in the retroperitoneal space is called renal ectopia. Crossed renal ectopia is a rare congenital malformation with an incidence ranging in births from 1/1350 to $1 / 2000$. Renal cell carcinoma is the most commonly reported tumor in renal fusion anomalies. Those anomalies increase the risk for neoplasia up to 4-fold.

CLINICAL CASE: A 64-year-old man had a past medical history of ectopic kidney and an oncologic pulmonary finding. Routine studies revealed a mass in the fusion of the two kidneys. Robotic-assisted laparoscopic isthmusectomy, ureteral repair with a Scardino flap, and double-J catheter placement were performed.
\end{abstract}

CONCLUSION: Crossed-fused renal ectopia is thought to be the abnormal development of the ureteral yolk and metanephric blastema during the fourth and eighth week of gestation. After horseshoe kidney, crossed-fused renal ectopia is the most common. Crossed ectopic kidney is a rare congenital alteration. The left-to-right cross is frequent, contrary to the case reported herein, and malignancy related to those anomalies is an exceptional event. The anatomy is unpredictable, and vascular abnormalities are observed in $70 \%$ of the cases, complicating surgical management. Different studies have attempted to establish the origin of the sarcomatoid phenotype and its relation to classic histologic patterns of renal carcinoma.

KEYWORDS: Crossed renal ectopia; Isthmusectomy; Kidney tumor

\footnotetext{
${ }^{1}$ Director.

${ }^{2}$ Fellowship en Endourología y Laparoscopia Avanzada.

${ }^{3}$ Urólogo.

${ }^{4}$ Fellowship en Cirugía Robótica.

Instituto de Urología Robótica y Laparoscópica, Nuevo León, Monterrey.
}

\section{Correspondence}

Dr. Juan Iván Briseño Rentería

dr_briseno@hotmail.com

\section{ANTECEDENTES}

La localización anómala del riñón en el espacio retroperitoneal se denomina ectopia renal. La ectopia renal congénita es una alteración durante el desarrollo embriológico del sistema genitourinario, en el que los riñones ascienden hasta ocupar su sitio normal en el retroperitoneo. $^{1}$
La ectopia renal cruzada constituye una malformación congénita excepcional, pues su incidencia va de 1 caso por cada 1350 a 1 caso por cada 2000 nacimientos, es decir, de 0.5 a $0.013 \%$ de los casos, con principal afectación en hombres que en mujeres (relación 2:1, respectivamente). ${ }^{2}$

El tumor más frecuentemente reportado en pacientes con anomalías de la fusión renal es 
el carcinoma de células renales, aunque su incidencia no es más alta que la reportada en la población sana. ${ }^{3}$

Otros tumores asociados con anomalías de la fusión renal incluyen: carcinoma de células transicionales, nefroblastoma (tumor de Wilms), oncocitoma, sarcoma, colesteatoma y teratoma. ${ }^{3}$

Mesrobian y sus colaboradores señalan que las anomalías de la fusión renal aumentan el riesgo de neoplasias puede ser cuatro veces mayor que en la población general. ${ }^{4} \mathrm{El}$ riesgo de sarcoma renal primario es poco frecuente, pues su incidencia es de $1-3 \%{ }^{4}$

\section{CASO CLÍNICO}

Paciente masculino de 64 años de edad, con antecedente de hipertensión arterial de 15 años de evolución (en tratamiento con losartan 50 mg/12 h) y ectopia renal cruzada derecha-izquierda fusionada en vigilancia. Un mes antes de acudir al servicio médico sufrió derrame pleural izquierdo; el estudio de imagen evidenció una masa de $2 \mathrm{~cm}$, se efectuó lobectomía y el resultado histopatológico fue sarcoma mixoide.

El paciente acudió a consulta externa, en donde refirió su antecedente de ectopia renal y el hallazgo pulmonar. En los estudios de rutina se encontró una masa en la fusión de ambos riñones, detectada por tomografía computada, además de percibir una masa en la fosa iliaca izquierda.

Los estudios de laboratorio reportaron: leucocitos $16.9 \times 10 / \mu \mathrm{L}$, eritrocitos $3.6 \times 10 / \mu \mathrm{L}$, hemoglobina $11.8 \mathrm{~g} / \mathrm{dL}$, hematocrito $33.1 \%$, plaquetas $598 \times 10 / \mu \mathrm{L}$, glucosa $92 \mathrm{mg} / \mathrm{dL}$, creatinina $0.7 \mathrm{mg} / \mathrm{dL}$, urea $40.7 \mathrm{mg} / \mathrm{dL}$, calcio 9.7 $\mathrm{mg} / \mathrm{dL}$, albúmina 3.4, TGO $23 \mathrm{U} / \mathrm{L}$, TGP $34 \mathrm{U} / \mathrm{L}$ y fosfatasa alcalina $103 \mathrm{U} / \mathrm{L}$.
La tomografía (UroTac) evidenció la ectopia renal cruzada derecha-izquierda, con fusión en el polo inferior izquierdo; en relación con la fusión del riñón derecho se observó una masa de 88 × 91 mm que obstruyó el sistema colector izquierdo, con discreta dilatación, identificación de un componente central compatible con necrosis, con características compatibles con algún proceso neoproliferativo (Figura 1).

La resonancia magnética de abdomen reportó la anomalía congénita del paciente, además de una masa sólida entre ambos riñones, de $90 \times$ 100 mm, con hidronefrosis en el riñón izquierdo; en la fase arterial se observó hipercaptación periférica de la tumoración y centro hipodenso (Figura 2).

Con base en los estudios radiológicos se estableció el diagnóstico de ectopia renal cruzada derecha-izquierda fusionada con tumor renal en el istmo.

Se programó la istmectomía laparoscópica asistida por robot, que se llevó a cabo en 270 minutos, con pérdida estimada de sangre de 1200 cc. Durante el procedimiento quirúrgico se seccionó el uréter del riñón izquierdo, debido a la invasión de esta estructura en el tercio medio,

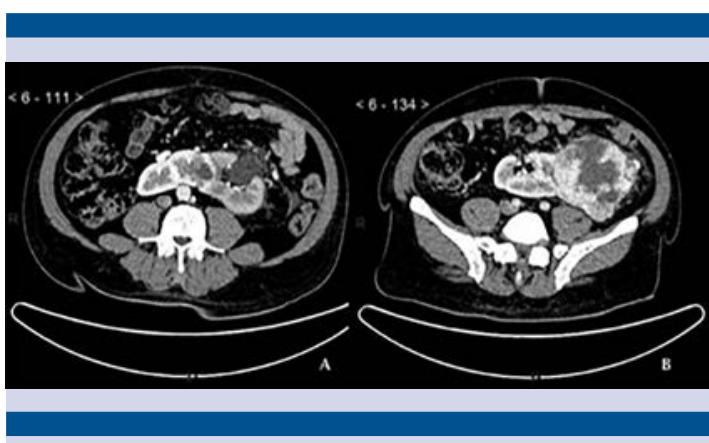

Figura 1. UroTac. Ectopia renal cruzada de derecha a izquierda con fusión en el polo inferior izquierdo (A) y en relación con la fusión del riñón derecho se observa una masa $88 \times 91 \mathrm{~mm}$. 


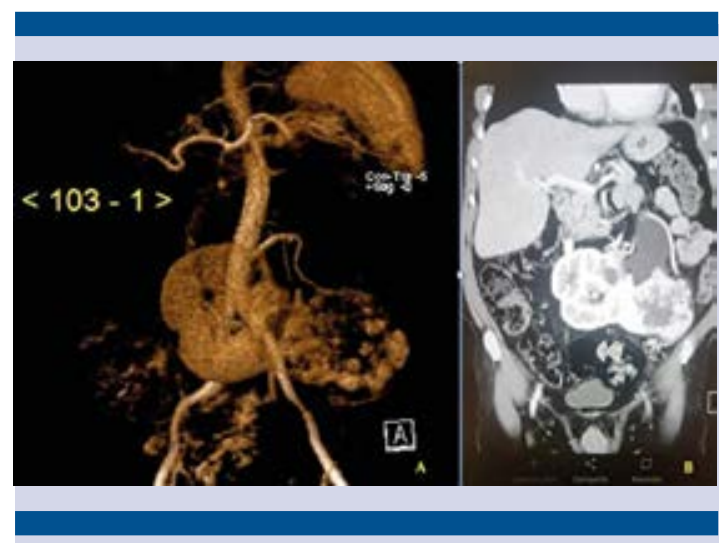

Figura 2. Resonancia magnética de abdomen que muestra la anomalía congénita (A) y la masa sólida entre ambos riñones, de 90 x 100 mm, con hidronefrosis en el riñón izquierdo (B).

por lo que se efectuó la plastia ureteral con colgajo de Scardino-Prince, colocación de catéter doble j y extracción de la pieza quirúrgica. El paciente se mantuvo en buenas condiciones, con recuperación satisfactoria.

El estudio histopatológico reportó: pieza de nefrectomía parcial de $466 \mathrm{~g}$, de $11.5 \times 10$ x $9 \mathrm{~cm}$. Histológicamente se clasificó como carcinoma de células claras Fuhrman II, que correspondió a $40 \%$ de la neoplasia; el resto del tejido correspondió a sarcoma pleomórfico, con inmunohistoquímica positiva para vimentina.

\section{DISCUSIÓN}

La ectopia renal cruzada fusionada puede originarse por el desarrollo anormal de la yema ureteral y el blastema metanéfrico durante la cuarta y octava semanas del embarazo. Después del riñón en herradura, la ectopia cruzada fusionada es la malformación renal más frecuente. En 1938, Wilmer clasificó inicialmente las anomalías de la fusión renal; sin embargo, en 1957 McDonald y McClellan incluyeron la ectopia cruzada en esta clasificación. 5,6
La ectopia renal cruzada es una alteración congénita poco frecuente, que en la mayoría de los casos suele aparecer con la fusión de ambos riñones. La incidencia en autopsias se ha reportado en 1 por cada 2000 pacientes, con mayor predominio en hombres que en mujeres $(3: 2)$, con el cruce de izquierda a derecha, contrario a nuestro caso, que lo hace una manifestación excepcional. La anomalía más común implica la fusión entre el polo del riñón ortotópico y el polo superior del riñón ectópico. ${ }^{5,7}$ La relación entre tumores renales malignos y ectopia renal cruzada fusionada es un evento extremadamente raro. $^{5}$

Las alteraciones anatómicas son impredecibles; se observan anomalías vasculares en $70 \%$ de los casos, el cuidado preoperatorio incluye una buena planificación quirúrgica con tomografía computada (TC), resonancia magnética (RM) o angiografía por TC/RM. ${ }^{5}$

Diversos estudios han intentado establecer el origen del fenotipo sarcomatoide y su relación con cualquier patrón histológico del carcinoma renal. En esta línea, los análisis comparativos de inactivación del cromosoma X en pacientes con carcinoma de células claras (subtipo más común de carcinoma renal) y con patrón sarcomatoide sugieren un origen común a partir del mismo tipo celular; diferentes patrones de pérdida alélica en distintas regiones cromosómicas parecen asociarse con la heterogeneidad y divergencia genética en el proceso de evolución de los distintos patrones, lo que origina los distintos fenotipos conocidos. ${ }^{8}$

El término de carcinosarcoma se refiere a la coexistencia de elementos heterólogos en el seno de un carcinoma, neoplasias con alta tasa de malignidad local y elevado grado de metástasis, para el que desafortunadamente no existe un tratamiento específico estandarizado; sin embargo, la combinación de cirugía y quimio- 
terapia constituye el protocolo de elección más indicado, ${ }^{8}$ similar al que se utilizó en el paciente de este estudio.

En pacientes con metástasis pulmonar sincrónica, sin enfermedad extrapulmonar, el tratamiento de elección es la quimioterapia. En casos escrupulosamente seleccionados, la cirugía por metástasis de respuesta puede indicarse como una opción después de la evaluación multidisciplinaria, teniendo en cuenta su sitio e historia natural de la enfermedad de cada caso. ${ }^{9}$

La cirugía, ablación o radioterapia de metástasis extrapulmonares puede ser una alternativa sin quimioterapia en pacientes con liposarcoma mixoide o tumor fibroso solitario. ${ }^{9}$ En nuestro paciente se decidió efectuar el procedimiento quirúrgico con quimioterapia coadyuvante.

Este es el primer reporte en México de un caso de ectopia renal cruzada fusionada y su tratamiento quirúrgico.

\section{CONCLUSIÓN}

La ectopia renal cruzada fusionada es una alteración excepcional asociada con anomalías de la vía genitourinaria superior, incluso la heterogeneidad de la manifestación histopatológica.
Esta alteración supone un pronóstico sombrío a corto y mediano plazo.

\section{REFERENCIAS}

1. Aguilera-Tubet C, Del Valle-Schaan JI, Martín-García BI. Tumor renal en ectopia renal cruzada con fusión. Actas Urol Esp 2005;29(10):993-996.

2. Castillo AO, Sánchez-Salas R, Foneron A, Vitagliano G. Heminefrectomía laparoscópica en ectopia renal cruzada con fusión inferior de unidades renales. Arch Esp Urol. 2010;63(1):58-61.

3. Stimac G, Dimanovski J, Ruzic B, et al. Tumors in kidney fusion anomalies report of five cases and review of the literature. Scand J Urol Nephrol. 2004;38:485-489.

4. Bergerat S, Barthelemy P, Mouracade P. Report Primary CIC-DUX4 round cell sarcoma of the kidney: A treatment refractory tumor with poor outcome. Pathol Res Pract. 2017. 213(2);154-160.

5. Ozgur Cakmak, Cemal Selcuk Isoglu, Ercument Aziz Peker. Renal cell carcinoma in patient with crossed fused renal ectopia. Archivio Italiano di Urologia e Andrologia 330 2015; 87, 4.

6. Solanki S, Bhatnagar V, Gupta AK, Kumar R. Crossed fused renal ectopia: Challenges in diagnosis and management. J Indian Assoc Pediatr Surg 2013;18:7-10.

7. Reza MN, Ghorbani H, Amin E, Arbab A, Ghadian A. Unusual Presentation of Renal Cell Carcinoma in Crossed Ectopic Kidney. Nephro Urol Mon. 2015 ; 7(3): e26760.

8. Torres-Gómez FJ, Torres-Olivera FJ. Carcinoma sarcomatoide renal con elementos heterólogos malignos a propósito de un caso. Arch. Esp. Urol. 2006. 59; 6:621-624.

9. The ESMO/European Sarcoma Network Working Group, Soft tissue and visceral sarcomas: ESMO Clinical Practice Guidelines for diagnosis, treatment and follow-up. Annals Oncol. 2014.25 (Supplement 3):102-112. 